\title{
Orientação para o Mercado no Segmento Hoteleiro: o caso português
}

\author{
Orientation for the Hotel Segment Market: the portuguese case
}

\author{
Cristina Maria Santos Estevão ${ }^{1}$ \\ Emerson Wagner Mainardes ${ }^{2}$ \\ Mario Lino Barata Raposo ${ }^{3}$
}

\begin{abstract}
Resumo
O objectivo deste estudo foi medir o grau de orientação para o mercado dos maiores grupos hoteleiros de Portugal. Após uma breve explanação sobre a orientação para o mercado e o segmento hoteleiro em Portugal apresentou-se um estudo empírico realizado junto dos maiores 20 grupos de hotéis de Portugal, utilizando como instrumento de colecta de dados, a tradicional escala Markor (Market orientation) adaptada ao sector hoteleiro. Constatou-se nas organizações investigadas uma boa capacidade de geração de informações de mercado e resposta ao mercado. Porém os resultados obtidos com o constructo da disseminação de informações de mercado ficaram abaixo das expetactivas. Portanto, o desafio actual para as principais redes de hotéis de Portugal é propiciar a melhoria da disseminação interna das informações que os profissionais de marketing recolhem junto ao mercado.

Palavras-chave: gestão do marketing; orientação para o mercado; markor; sector hoteleiro em Portugal.
\end{abstract}

\begin{abstract}
The objective of this study was to measure level of orientation for the market of the largest hotel groups of Portugal. After a brief explanation on the market orientation and the hotel segment in Portugal an empiric study was presented performed with the largest groups of 20 hotels of Portugal, using as an instrument of collection data, the traditional Markor scale (market orientation) adapted to the hotel sector. It was found in the investigated organizations a good capacity to generate market information and response to the market. However the results obtained with the construct of the dissemination of market information were below the expectations. So the current challenge for the main networks of hotels in Portugal is to improve the internal dissemination of information that marketing professionals gathered at the market.
\end{abstract}

Keywords: marketing management, orientation to the market, markor, hotel sector in portugal.

\footnotetext{
${ }^{1}$ Doutoranda em Gestão pela Universidade da Beira Interior (UBI) - Covilhã/Portugal; Mestre em Gestão pela Universidade da Beira Interior (UBI); Professora da Estratégia e Marketing; Investigadora em Turismo, Estratégia, Competitividade, Inovação e Marketing. E-mail: kristina.estevao@hotmail.com

2 Doutorando em Gestão pela Universidade da Beira Interior (UBI) - Covilhã/Portugal; Mestre em Administração pela Universidade Regional de Blumenau (FURB); Professor de Marketing; Pesquisador do Núcleo de Estudos em Ciências Empresariais (NECE); Investigador em Gestão Universitária e Marketing Educacional. E-mail: emerson.wm@sapo.pt

${ }^{3}$ Doutor em Gestão pela Universida de Barcelona (UB); Professor Catedrático do Departamento de Gestão e Economia da Universidade da Beira Interior (UBI); Professor de Marketing, Estratégia e Empreendedorismo; Coordenador Cientifico do Núcleo de Estudos em Ciências Empresariais (NECE); Investigador em Marketing, Estratégia e Empreendedorismo; Vice-presidente para Portugal do ECSB European Council of Small Business. E-mail: mraposo@ubi.pt
} 


\section{Introdução}

O despertar do consumo trouxe mudanças de ordem econômica, política, social, onde anteriormente imperavam as formas de produção e gestão de pequena escala, típicas dos séculos XVIII e XIX. Com a produção em massa para atender às necessidades crescentes dos consumidores de todo o globo, apareceu o marketing dentro das organizações (KOHLI; JAWORSKI, 1990), afinal a primeira função de qualquer empresa é manter a sua actividade, sendo desta forma, necessário gerar e manter consumidores (LEVITT, 1986).

Nas duas primeiras décadas do século XX, o marketing tinha como função a facilitação do comércio e distribuição de produtos (BARTELS, 1988). A partir de 1920, o marketing passa a tentar compreender os motivos que levavam os consumidores a adquirirem produtos e serviços, algo que se solidifica somente a partir dos anos 40 .

Daí em diante, passou a ser fundamental para as empresas a implementação do marketing não somente como mais uma função organizacional, mas principalmente como uma filosofia que abrange toda a empresa, em todos os níveis hierárquicos (NARVER; SLATER, 1990). Actualmente, não é exagero afirmar que a gestão do marketing é a função mais importante nas organizações (JOCZ; QUELCH, 2008).

Segundo Kotler e Armstrong (1996), actualmente a gestão do marketing nas empresas tem o intuito de satisfazer os seus consumidores, planear produtos, serviços e programas adequados, determinando as acções das organizações no mercado, sendo as demais áreas das empresas subordinadas às decisões dos gestores de marketing, afinal atender as necessidades e desejos dos clientes tornou-se fundamental para a sobrevivência e crescimento de qualquer organização no mercado, facto este que justifica estudos a respeito do tema.

Entre as organizações do mercado, as empresas do setor hoteleiro destacam-se por serem importantes para o desenvolvimento turístico de qualquer nação, inclusive Portugal, importante destino turístico europeu. Neste país, o turismo cresce ano a ano e ganhou relevante representatividade na economia portuguesa. Sendo um setor estratégico para esta nação, investigar a orientação para o mercado dos hotéis presentes em Portugal, elemento fundamental para o desenvolvimento do turismo, mostra-se importante para ações que incentivam o turismo em solo português.

Sendo assim, questiona-se - Qual é o grau de implementação do conceito de marketing nos principais grupos hoteleiros de Portugal? Para responder a esta questão, este estudo teve como objectivo geral medir o grau de orientação para o mercado das maiores redes de hotéis 
de Portugal. Como objectivos específicos, buscou-se: (a) comparar o grau de geração e disseminação de informações de marketing e a resposta ao mercado entre os hotéis de origem portuguesa versus hotéis de origem estrangeira; (b) comparar o grau de geração e disseminação de informações de marketing e resposta ao mercado entre os profissionais de marketing dos hotéis portugueses versus profissionais das mesmas organizações, mas que actuam em departamentos não ligados ao marketing; (c) identificar os elementos que podem ser aperfeiçoados para a optimização dos graus de geração e disseminação de informações de marketing e de resposta ao mercado dos grandes grupos de hotéis presentes em Portugal.

O estudo em questão pretendeu contribuir para com as empresas do segmento hoteleiro português, ao indicar o grau de orientação de mercado dos principais representantes do sector. As contribuições estendem-se aos praticantes do marketing, ao estabelecer a importância da implementação do conceito de marketing para todas as organizações, e para a academia, ao demonstrar a importância dos estudos em marketing para o meio organizacional e para a sociedade em geral.

Para a realização deste estudo, o marketing foi contextualizado como uma função da empresa e como uma filosofia de gestão. De seguida, descreveu-se a orientação para o mercado e os modelos desenvolvidos para medir o grau de orientação para o mercado nas organizações posicionando-os actualmente. Após uma breve caracterização do estado actual do sector hoteleiro português, seguiu-se a apresentação dos métodos e técnicas de pesquisa utilizados. Em seguida, apresentou-se a análise dos dados coletados. Finaliza-se o estudo com as conclusões e implicações do estudo realizado, bem como as recomendações de linhas futuras de investigação de continuidade a este estudo.

\section{O Marketing como Filosofia de Gestão das Organizações}

Desde a sua concepção, o marketing tornou-se uma função empresarial, evoluindo com o tempo para uma filosofia empresarial. Desde a década de 60, a incapacidade de se distinguir entre as duas tem sido citado por vários autores (KOTLER; LEVY, 1969; HOWARD, 1983; LEVITT, 1986; BROWN, 1987; GRÖNROOS, 1989; McKENNA, 1991; KOTLER; ARMSTRONG, 1996; BAKER, 2003; KOTLER, 2005; WILKIE; MOORE, 2007), como um dos factores essenciais na promoção da incompreensão sobre a natureza do marketing e o seu papel na gestão empresarial (BROWN, 1987). 
Para Kotler e Armstrong (1996), a gestão do marketing numa organização não limita-se a identificar as necessidades dos consumidores da empresa, mas também envolve o planeamento de produtos, serviços e programas adequados. Ou seja, não é uma função isolada na organização, mas sim um orientador de todas as acções da empresa, bem como um agente propulsor de associações externas à organização com vistas a proporcionar um valor superior aos seus consumidores. O marketing na verdade é um trabalho de todos na empresa (McKENNA, 1991).

Este papel do marketing dentro das organizações foi objecto de estudo de Brown (1987). Para o autor, o verdadeiro papel do marketing na organização, na realidade, é agir como uma ponte entre as várias funções da empresa e como eles interpretam o seu papel, e como vêem as necessidades dos clientes da empresa. O autor concluiu que o marketing tem a tarefa de compreender as necessidades do cliente e de comunicar e orientar as suas descobertas para as demais áreas da empresa, a fim de garantir que todas se tornem verdadeiramente orientadas para os clientes, conforme pode ser visualizado na figura 1, que apresenta o modelo proposto por Brown (1987).

Convém salientar que o marketing não é apenas a procura cega de qualquer cliente a qualquer preço, uma interpretação errônea e muito comum entre os profissionais que não compreendem o marketing (BAKER, 2003). Os gestores funcionais não devem sentir que as implicações do marketing são de que devem satisfazer todos os potenciais clientes, independentemente do custo. O marketing envolve a identificação e prossecução de objectivos claramente definidos pelos clientes, aqueles em que a empresa seja a mais adequada para servir num mercado concorrencial (BROWN, 1987). 


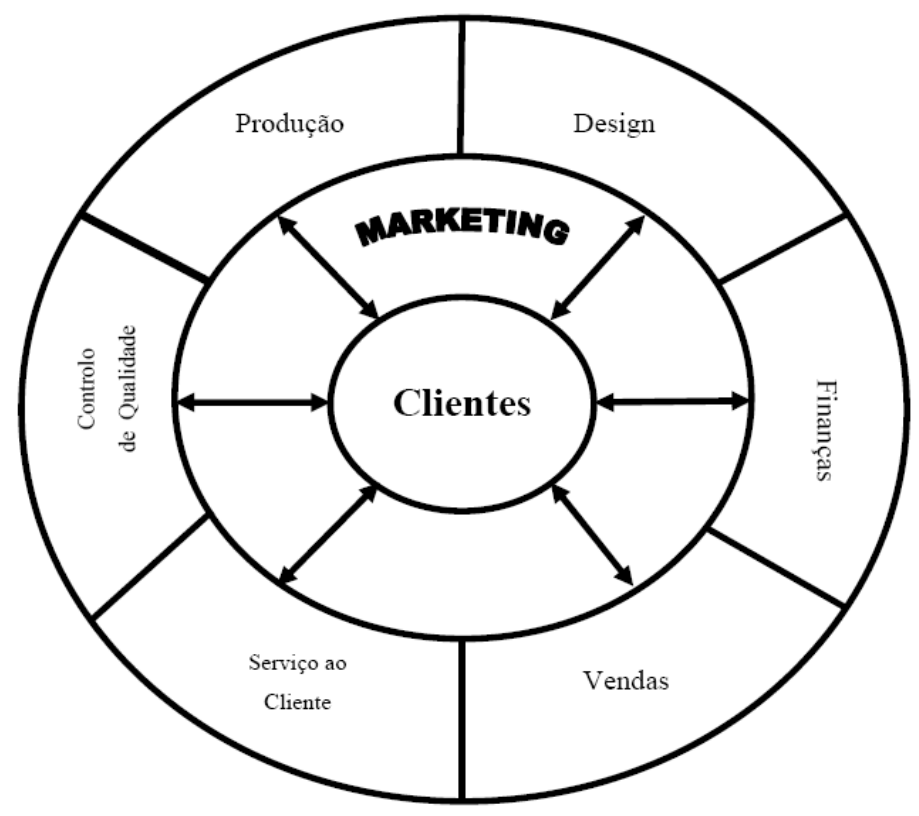

Figura 1 - Papel do Marketing nas Organizações

Fonte: Adaptado de Brown (1987, p. 27)

Para Thomas (2006), o marketing como uma cultura organizacional, preserva de modo uniforme toda a empresa, o activo mais importante de uma organização - o seu relacionamento com os seus clientes globais. Já segundo Greenley, Hooley e Saunders (2004), os profissionais de marketing têm um papel crítico como defensores internos dos clientes e para um sistema de valor que põe o cliente em primeiro lugar, afinal o recurso mais importante de qualquer empresa é o seu público consumidor, algo que deve estar presente na mente de todos os colaboradores da organização, independente da sua função.

Conhecendo e interpretando-se as necessidades dos actuais e potenciais consumidores dos produtos e/ou serviços de uma organização, é possível estabelecer políticas e planos, desenvolver competências, orientar recursos e sectores da empresa, ou seja, focalizar todas as acções da organização para as necessidades e desejos dos clientes, aumentando a probabilidade de sucesso da empresa (PEARSON, 2002). A gestão de todo este processo é liderada pelos profissionais do marketing (KOTLER; KELLER, 2005).

Porém, para que seja possível a gestão focada no cliente é necessário que o conceito de marketing seja implementado em toda a organização. Esta implementação é tradicionalmente conhecida como orientação para o mercado (KOHLI; JAWORSKI, 1990). 


\section{Organizações Orientadas para o Mercado}

A evolução do conceito de marketing, tendo como ponto principal o cliente no centro da estratégia empresarial, fez com que as organizações passassem a ter uma orientação estratégica voltada para o mercado (KOTLER; ARMSTRONG, 1996).

O termo “orientação para o mercado” surgiu como uma cultura a nível organizacional, isto é, como um conjunto de valores e crenças que coloca o consumidor em primeiro lugar, no momento da elaboração da estratégia da organização (DESHPANDÉ; WEBSTER, 1989). No início da década de 90, muitas definições e modelos surgiram acerca deste conceito, nomeadamente através dos trabalhos desenvolvidos por Kohli e Jaworski (1990), Narver e Slater (1990), Desphandé, Farley e Webster (1993) e Day (1994) e que permanecem válidos até hoje. Neste estudo optou-se pelo modelo proposto por Kohli e Jaworski (1990). A escolha deveu-se ao fato de ser o modelo mais utilizado e validado pelos investigadores que pesquisam a orientação para o mercado.

\subsection{Orientação para o mercado segundo Kohli e Jaworski (1990)}

Kohli e Jaworski (1990) definiram o conceito de orientação para o mercado como sendo a geração da informação do mercado para toda a organização relativa às necessidades actuais e futuras dos clientes, a disseminação da informação do mercado através dos departamentos e a resposta da empresa a esta informação. O estudo destes autores sobre a orientação para o mercado identificou três componentes: i) geração de informação, ii) disseminação de informação e iii) resposta ao mercado.

A geração de informação de mercado relaciona-se com factores exógenos à organização, em que todos os departamentos devem recolher e tratar a informação acerca das necessidades e exigências actuais e futuras dos clientes e também sobre factores que possam atingir directamente os desejos dos consumidores, nomeadamente concorrentes, fornecedores, factores político-tecnológicos, sócio-culturais, entre outros, de forma a organização poder monitorizar a conjuntura e o ambiente externo.

Com a informação recolhida, esta deve ser difundida pelos vários sectores da organização para que seja conhecida e partilhada no seio da organização, sendo esta a visão apresentada para a disseminação da informação. Posteriormente, aos dois primeiros componentes surge a construção da resposta ao mercado que consiste na formulação e execução de acções lógicas com o estudo do mercado, isto é, consiste na transformação do conhecimento adquirido nas 
etapas anteriores em acções reais, que irão reflectir-se nas necessidades e desejos actuais e futuros dos consumidores reais e potenciais.

Segundo Kohli e Jaworski (1990), uma organização orientada para o mercado implementa e age de acordo com o conceito de marketing, ou seja, é aquela cujas acções são consistentes com o conceito de marketing. A proposta inicial dos autores resultou numa escala, conhecida como Markor (Market orientation), que foi proposta inicialmente com 3 construtos e 32 variáveis (geração de inteligência de marketing - 10 variáveis, disseminação de inteligência de marketing - 8 variáveis, e resposta ao mercado - 14 variáveis) e que mede o grau de orientação para o mercado de uma determinada organização.

Esta escala foi testada (JAWORSKI; KOHLI, 1993) e posteriormente ajustada para 20 variáveis e validada estatisticamente (KOHLI; JAWORSKI; KUMAR, 1993), sendo a escala Markor a mais utilizada por acadêmicos e praticantes do marketing quando se busca encontrar o grau de implementação do conceito de marketing em toda a organização, por outras palavras, a orientação da empresa para o seu mercado (KOLLER, 2002).

\subsection{Orientação para o mercado no século XXI}

Segundo Foley e Fahy (2009), a operacionalização do conceito da orientação para o mercado de investigadores como Kohli e Jaworski (1990) e Narver e Slater (1990) teve um benefício significativo para a progressão do pensamento do marketing, influenciando-o até os dias actuais.

Ainda hoje, os antecedentes sobre a orientação para o mercado representam importantes alavancas para aumentar a orientação dentro de uma empresa no mercado. O conhecimento destes antecedentes ajuda certamente aos gestores nos seus esforços para implementar uma orientação para o mercado (RAIIJ; STOELHORST, 2008).

Apesar dos principais estudos sobre a orientação para o mercado serem da década de 90, actualmente esta temática continua a merecer grande atenção dos investigadores, apesar de não surgirem novos modelos que suplantem os modelos desenvolvidos nos anos 90. Foi o caso de Lafferty e Hult (2001), que deram uma importância fundamental à definição dos conceitos sobre a orientação do mercado e afirmaram que numerosos projectos de investigação têm definido os constructos e explorado a sua aplicação e implementação nas empresas. Com o tempo, a orientação do mercado tornou-se sinônimo de como implementar o conceito de marketing. 
Lafferty e Hult (2001) apresentaram um quadro que reuniu conceituações contemporâneas da orientação de mercado a partir de uma síntese dos seus componentes. Embora existam algumas diferenças entre os modelos, a ênfase de orientação para o mercado está em satisfazer as necessidades e criar valor para o cliente. Um segundo elemento comum é a importância da informação dentro da organização. Esta informação é tudo o que pode ser gerado sobre os clientes e os concorrentes, para ajudar na busca da empresa para o mercado para o qual está orientado. Logo que esta informação estiver acessível, através do esforço concertado de todas as diferentes funções dentro da empresa, a organização deve, em seguida, divulgar esse conhecimento a todas as unidades estratégicas de negócios da organização e dos seus departamentos. Esta interfunctional coordenação é o terceiro princípio unificador nos modelos. Finalmente, as quatro perspectivas sobre a orientação do mercado sublinham a necessidade de tomar medidas adequadas por parte da empresa para implementar as estratégias necessárias para o mercado que ela busca.

Considerando a importância deste construto para o desempenho da organização e do seu potencial para fornecer uma vantagem competitiva, é provável que a conceituação de orientação do mercado continue a evoluir, porém seguindo os princípios desenvolvidos na última década do século XX (LAFFERTY; HULT, 2001).

\section{O Segmento Hoteleiro de Portugal}

Cooper et. al. (2005) afirmaram que o segmento hoteleiro é o maior sector dentro da economia turistica, sendo uma estrutura de apoio importante para um destino turistico. A hotelaria também pode surgir como um elemento importante em estratégias mais amplas de desenvolvimento económico.

O relatório PITER (2005) reafirma que a hotelaria é um sector de suporte de base ao apoio turístico, sendo muito importante no desenvolvimento da indústria do turismo. É de realçar o papel que o alojamento tem na economia local (uma vez que é ao nível deste sector que são efectuadas as maiores despesas) ajudando positivamente para o aumento dos rendimentos das populações locais (efeito multiplicador).

Em Portugal, segundo o Atlas da Hotelaria 2009 (2009), a oferta de alojamento compreendia 1.209 estabelecimentos hoteleiros até à data de 31 Dezembro de 2008, que inclui aldeamentos turísticos, apartamentos turísticos, hotéis, hotéis apartamentos e pousadas de Portugal. 
Tabela 1 - Ranking dos Hotéis em Portugal

\begin{tabular}{|c|c|c|c|c|c|c|c|}
\hline \multirow[b]{2}{*}{ 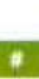 } & \multirow[b]{2}{*}{ Grupo Hoteleiro / Entidade de Management } & \multicolumn{2}{|c|}{ Estabelecimentos Hoteleiros } & \multicolumn{2}{|c|}{ Unidades de Alojamento' } & \multicolumn{2}{|c|}{ Camas } \\
\hline & & Numero & $\%$ do Total & Número & \% do Total & Numero & \% do Total \\
\hline 1 & Pestana Hotels \& Resorts / Pestana Pousadas & 64 & $5.3 \%$ & 4.677 & $4.4 \%$ & 9278 & $3.8 \%$ \\
\hline 2 & Vila Gale Hoteis & 14 & $1.2 \%$ & 3.191 & $3.0 \%$ & 6.442 & $2.7 \%$ \\
\hline 3 & Accor Hotels & 28 & $2,3 \%$ & 2.945 & $2,7 \%$ & 5.374 & $2,2 \%$ \\
\hline 4 & Espinto Santo Hoteis & 10 & $0.8 \%$ & 2393 & $2.2 \%$ & 4.912 & $2.0 \%$ \\
\hline 5 & VIP Hotels & 13 & $1.1 \%$ & 2,309 & $2,2 \%$ & 3.414 & $1.4 \%$ \\
\hline 6 & Grupo Hotti & 14 & $1.2 \%$ & 1877 & $1,8 \%$ & 4273 & $18 \%$ \\
\hline 7 & Starmood Hotels \& Resorts & 6 & $0.5 \%$ & 1.789 & $1,7 \%$ & 4.108 & $1.7 \%$ \\
\hline 8 & Lunahoteis & 16 & $1.3 \%$ & 1.693 & $1,6 \%$ & 4649 & $1.9 \%$ \\
\hline 9 & Grupo Iberatel & 5 & $0.4 \%$ & 1.643 & $1.5 \%$ & 4.740 & $2.0 \%$ \\
\hline 10 & Dom Pedro Hotels & 7 & $0.6 \%$ & 1.399 & $1,3 \%$ & 2915 & $1.2 \%$ \\
\hline 11 & Continental Group & 12 & $1.0^{\mathrm{L} \%}$ & 1.347 & $1.3 \%$ & 2.195 & $0.9 \%$ \\
\hline 12 & Sana Holels & 9 & $0,7 \%$ & 1.275 & $1.2 \%$ & 2066 & $0.9 \%$ \\
\hline 13 & Riu Hotels \& Resorts & 3 & $0.2 \%$ & 1.176 & $1,1 \%$ & 2352 & $1.0 \%$ \\
\hline 14 & Martiott & 4 & $0,3 \%$ & 1.139 & $1,1 \%$ & 1.967 & $0,8 \%$ \\
\hline 15 & Porto Bay Hoteis \& Resorts & 6. & $0,5 \%$ & 1.099 & $1.0 \%$ & 2.198 & 0,996 \\
\hline 16 & Hoteis Real & 7 & $0.6 \%$ & 1.087 & $1.0 \%$ & 2.245 & $0.9 \%$ \\
\hline 17 & Hoteis Fènix & 7 & $0.6 \%$ & 1.062 & $1.0 \%$ & 2030 & $0.8 \%$ \\
\hline 18 & Bensaude Tunsmo & 8 & $0,7 \%$ & 1.058 & $1.0 \%$ & 2.074 & $0,9 \%$ \\
\hline 19 & Grupo Hoteleiro Fernando Barata & 6 & $0.5 \%$ & 886 & $0.8 \%$ & 1,692 & $0.7 \%$ \\
\hline \multirow[t]{5}{*}{20} & Peichey Leisure & 3 & $0.2 \%$ & 875 & $0.8 \%$ & 2.984 & $1.2 \%$ \\
\hline & Sub-total & 242 & $20.0 \%$ & 34920 & $32.6 \%$ & 71.908 & $29.8 \%$ \\
\hline & Outros Grupos Hoteleiros & 305 & $25.2 \%$ & 31.086 & $29,0 \%$ & 67.190 & $27.8 \%$ \\
\hline & Independentes & 652 & $54,8 \%$ & 41.165 & $38,4 \%$ & 102.205 & $42,4 \%$ \\
\hline & Total & 1.209 & $100 \%$ & 107.171 & $100 \%$ & 241.303 & $100 \%$ \\
\hline
\end{tabular}

Fonte: Atlas da Hotelaria 2009 (2009, p. 32)

No referido atlas foi apresentado um ranking dos 20 principais grupos hoteleiros (tabela 1), onde a base do ranking foi o número total de unidades de alojamento (número de quartos e apartamentos). Observando-se a tabela 1, constata-se que o Grupo Pestana (designado por Pestana Hotels \& Resorts / Pestana Pousadas) lidera o ranking em Portugal. No $2^{\circ}$ e $3^{\circ}$ lugar deste ranking surgem, respectivamente, Vila Galé Hotéis e Accor Hotels. Os grupos Espírito Santo Hotéis e Vip Hotels completam o Top 5 da Hotelaria em Portugal. Percebe-se também que os grupos hoteleiros presentes no Top 20 representam, juntos, 32,6\% da oferta nacional disponível de unidades de alojamento, sendo os demais 67,4\% dos alojamentos dividido entre 305 estabelecimentos pertencentes a pequenos grupos hoteleiros e 662 hotéis independentes. Isto leva a concluir que os 20 maiores grupos hoteleiros em Portugal são altamente significativos para o turismo no país. Portanto, uma forte orientação para o mercado destes grupos hoteleiros fomenta o importante mercado turístico português, beneficiando economicamente as comunidades locais, refletindo-se positivamente na economia de Portugal como um todo. 


\section{Métodos e Técnicas de Pesquisa}

A pesquisa de campo realizada, de caráter quantitativo-descritivo e corte transversal (HAIR et. al., 2003) teve como finalidade aplicar o tradicional modelo Markor, proveniente dos estudos de Kohli e Jaworski (1990), Jaworski e Kohli (1993) e Kohli, Jaworski e Kumar (1993), ao segmento hoteleiro português. Esta escala genérica mede a orientação para o mercado de uma determinada organização, independente do que esta produza.

A escala de medição da orientação de uma empresa para o mercado reflete o grau actual da implementação do conceito de marketing na organização. O desenvolvimento da escala Markor surgiu da necessidade de evidenciar a prática do marketing e a sua gestão nas organizações do mercado, ou seja, o modelo proposto por Kohli e Jaworski (1990), posteriormente validado e ajustado empiricamente (JAWORSKI; KOHLI, 1993; KOHLI; JAWORSKI; KUMAR, 1993), identifica, em três construtos, as acções organizacionais orientadas para o seu mercado-alvo: a geração das informações de mercado (inteligência de marketing); a disseminação destas informações por toda a organização; e, baseado nas informações levantadas e disseminadas, a resposta ao mercado com produtos e/ou serviços adequados às necessidades e desejos dos seus clientes.

A escala Markor, originalmente de 1990, foi inicialmente proposta com 32 variáveis em 3 construtos (geração de informações, disseminação de informações, resposta ao mercado). Posteriormente, em 1993, foi ajustada, mantendo os três construtos, porém medidos por 20 variáveis, todas já testadas e validadas empiricamente (KOHLI; JAWORSKI; KUMAR, 1993).

No caso específico deste estudo, buscou-se avaliar a implementação do conceito de marketing no segmento hoteleiro de Portugal, população-alvo desta investigação. Para isto, mediu-se, como amostra que representa de forma significativa este sector específico, a orientação para o mercado dos 20 maiores grupos de hotéis do país, conforme o ranking apresentado no Atlas da Hotelaria 2009 (2009), utilizando-se para isto a escala Markor mais recente, a versão de 1993. Este tipo de amostra configura-se como uma amostragem não-probabilística por julgamento, pois buscou-se realizar a investigação junto a elementos representativos qualitativamente de um determinado sector (HAIR et. al., 2003).

A escolha do segmento hoteleiro justifica-se por ser um importante segmento para a economia portuguesa; ser um dos sectores onde há maior empregabilidade e ser um dos principais 
segmentos a atrair recursos externos e investimentos para o país. Desta forma, escolheu-se os maiores grupos de hotéis que possuem unidades em solo português, bem como delimitou-se a investigação para a orientação para o mercado português nestes grupos, pois vários deles são filiais de grupos estrangeiros. Nestes grupos foram inquiridos executivos chefes e profissionais actuantes das principais áreas de um grupo hoteleiro (Marketing, Comercial, Compras, Materiais, Alojamentos, Cobranças, Grupos, Reservas, Corporativo, Eventos e Direção Geral), seguindo a mesma estratégia adoptada Kohli, Jaworski e Kumar (1993).

O inquérito aplicado pretendeu responder a uma série de questões: Qual o grau de geração de informações de marketing nas grandes redes de hotéis de Portugal? Qual o grau de disseminação de informações de marketing nestes grupos hoteleiros? Qual o grau de resposta ao mercado destes grupos? Quais os elementos que podem ser aperfeiçoados para a optimização dos graus de geração e disseminação de informações de marketing e de resposta ao mercado dos grandes grupos de hotéis presentes em Portugal?

Para responder a estas questões, os dados foram colectados por intermédio de um questionário seguindo os preceitos da escala Markor de 20 variáveis (KOHLI; JAWORSKI; KUMAR, 1993). A tradução da escala para o português foi anteriormente elaborada e validada por Koller (2002). Desta forma, construiu-se um questionário estruturado, não disfarçado (HAIR et. al., 2003), contendo afirmações seguidas de uma escala Likert de 5 pontos (discordo totalmente a concordo totalmente), onde os inquiridos deveriam identificar o seu grau de concordância para cada afirmação apresentada. O instrumento de coleta de dados foi dividido em quatro partes (anexo):

- Parte 1: Caracterização do grupo hoteleiro (nome do grupo, cidade sede, número de unidades em Portugal, classificação, função no grupo hoteleiro do responsável pelo preenchimento do inquérito);

- Parte 2: Geração de informações de marketing (6 afirmações específicas, retiradas da escala Markor, e 1 afirmação geral - capacidade geral de gerar informações de marketing);

- Parte 3: Disseminação de informações de marketing (5 afirmações específicas, retiradas da escala Markor, e 1 afirmação geral - capacidade geral de disseminar informações de marketing);

- Parte 4: Resposta ao mercado (9 afirmações específicas, retiradas da escala Markor, e 1 afirmação geral - capacidade geral de responder ao mercado). 
Por ser um instrumento de colecta de dados já muitas vezes testado e validado, dispensou-se a etapa do pré-teste. A colecta de dados aconteceu no período de 11 a 26 de Maio de 2009. Foram enviados por email 60 questionários, três para cada rede presente no ranking dos 20 maiores grupos de hotéis em Portugal (um inquérito para o gestor do marketing da rede, dois inquéritos para gestores de outras áreas do grupo). Destes, 30 questionários retornaram preenchidos. Estes foram analisados individualmente e 28 foram considerados válidos.

Ao todo, 17 dos 20 principais grupos hoteleiros de Portugal participaram voluntariamente da investigação, com pelo menos um questionário preenchido. Em contrapartida, três grupos hoteleiros informaram que não tinham interesse em participar da pesquisa, não respondendo a nenhum dos três inquéritos enviados.

\section{Análise dos Resultados}

Com a finalização da coleta de dados, estes foram devidamente tabulados para a realização das análises. Para analisar os resultados utilizou-se análises descritivas básicas e análises multivariadas, especialmente a regressão linear múltipla (HAIR et. al., 2003), que permitiu identificar os elementos que podem ser aperfeiçoados para a optimização dos graus de geração e disseminação de informações de marketing e de resposta ao mercado dos grandes grupos de hotéis presentes em Portugal.

\subsection{Análises descritivas}

O primeiro passo na análise dos dados foi calcular as estatísticas básicas de cada constructo e variável. Para poder visualizar em detalhes a orientação para o mercado dos grandes grupos hoteleiros portuguesas, dividiu-se os resultados da seguinte forma: médias e desvio padrões de todos os grupos participantes da pesquisa; depois considerou-se somente grupos estrangeiros em território português; na seqüência somente grupos genuinamente portugueses; outra divisão foram os resultados obtidos exclusivamente dos profissionais de marketing para comparação com os profissionais de outras áreas das organizações investigadas. Os resultados obtidos podem ser observados na tabela 2. 
Tabela 2 - Estatísticas Descritivas

\begin{tabular}{|c|c|c|c|c|c|c|c|c|c|c|}
\hline Construtos $\theta$ Variavel 8 & Todos & Hoteis & Hoteis & Estrangeiros & Hoteis & Portugueses & Profissionais & de Marketing & Outros & Departamentos \\
\hline Geraça de informaços de Mercado & Tedra & Desvoraran & Thes/a & Desviopara & Thedla & Desvilo Padá & Tedla & Desilopadra & Theda & DeEVI Padá \\
\hline Enconiros periodlcos com cllentes & 4,32 & .77237 & 4,40 & ,894 & 4,30 & 767 & 4,44 & ,72ब & 4.26 &, 806 \\
\hline Pesquisa de mercado & 3,96 & ,96156 & 4,00 & 1,000 & 3,96 & 976 & 4,22 & ,833) & 3,89 & 1,049 \\
\hline Lentidâo na detecçăo de mudanças de preferenclas & 1,68 & 86297 & 1,20 & .447 & 1,78 & 902 & 1,56 & ,726 & 1,74 & ,933 \\
\hline Desquisa Junito a cilentes & 4,00 & 1,05409 & 4,00 & 1,000 & 4,00 & 1,087 & 4,67 & ,500 - & 3,79 & 1,134 \\
\hline Lent:dăo na detecçâo de mudanças no mercado & 1,75 & ,79931 & 1,20 & ,447] & 1,87 & ,815 & 1,56 & ,726 & 1,84 & ,834 \\
\hline Avaliaçăo periodica dos efeltos das mudanças no mercadoiclentes & 4,18 & 94491 & 4,60 & ,548 & 4,09 & 9966 & 4,56 & ,527] & 4,00 & 1,054 \\
\hline Capacidade geral de gerar intormaçbes do mercado & 4,07 &, 85758 & 4,60 &, 894 & 3,96 & 825 & 4.33 & .707 & 3.95 & ,911 \\
\hline 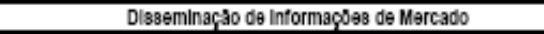 & \begin{tabular}{l|l} 
Tedla & \\
\end{tabular} & Desvo radra & Thedla & Desviopara & Thenla & Desvio Padra & Tedia & Deshopadra & Thela & Desणा० Padá \\
\hline Reunldes periodlcas entre departamentos & 4,32 & 90487 & 4,40 & ,894 & 4,30 & .926 & 4,78 & ,441 & 4,21 & ,976 \\
\hline Discussato MKTioutros deptos necessldades futuras dos cllentes & 4,07 & 1,11981 & 4,80 & .447 & 3,91 & 1,164 & 4,56 & ,726 & 3,79 & 1,182 \\
\hline Problemas com clentes Importantes transmilidas rapidamente a todos & 3,64 & 1,09593 & 4,80 & ,447] & 3,39 & 1,033 & 4,11 &, 782 & 3,32 & 1,108 \\
\hline Satistaçăo dos clentes dlvulgava regulamente em toda a empresa & 3,64 & 1,22366 & 5,00 & , coc & 3,35 & 1,152 & 4,11 & 1,167 & 3,37 ] & 1,165 \\
\hline Lent:daso de deptos se comunicarem sobre questides de mercado & 2,11 & 99403 & 1,60 & 894 & 2,22 & .998 & 1,78 & ,833 & 2,37 & 1,012 \\
\hline Capacidade geral de disseminaçăo de informaçes de mercado & 3,71 & 85449 & 4,20 & ,837 & 3,61 & .839 & 4.22 & ,833] & 3.53 &, 772 \\
\hline Resposta ao Mercado & \begin{tabular}{l|l} 
Tedla & \\
\end{tabular} & Desvo radra & Thedla & Desvio Para & Thedla & Desnloradra & Tesla & Desilopadra & Theola & Desilo Padra \\
\hline Lentida de resposia a concorrentes & 1,79 & 1,06657 & 2,00 & 1,732 & 1,74 & 915 & 1,44 &, 726 & 1,95 & 1,177 \\
\hline gnorar reconhecimento de novas necess/dades dos cllentes & 1,68 & 86297 & 1,20 &, 447 & 1,78 & 902 & 1,44 & ,726 & 1,79 & ,918 \\
\hline Revisto conthua dos servipos do hotel & 4,21 & ,83254 & 4,60 & ,548 & 4,13 & 869 & 4,56 & ,726 & 4,05 & 848 \\
\hline Reunldes de deptos para responder as mudanças no mercado & 4,04 & 83808 & 4,60 &, 548 & 3,91 & ,848 & 4,22 & ,833] & 3,84 & 834 \\
\hline Resposta a campanhas de comunicaç̧o de concorrentes & 3,50 & 1,23228 & 3,40 & 1,517 & 3,52 & 1,201 & 3,89 & 1,167 & 3,37 & 1,212 \\
\hline A:Midades coordenadas entre deptos & 3,61 & ,78595 & 4,60 &, 548 & 3,39 & ,656 & 4,00 & ,707 & 3,37] & 684 \\
\hline Redamaçbes dos clentes nâo sâo ouvidas & 1,46 & 1,17006 & 1,80 & 1,789 & 1,39 & 1,033 & 1,00 & , 000 & 1,47 & 1,124 \\
\hline Incapacidade de colocar em pratlca um grande plano de MKT & 1,64 & 86984 & 1,40 &, 548 & 1,70 & 926 & 1,22 & ,667] & 1,79 & ,918 \\
\hline Ao descobir necessldade de mudanças os deptor realzam-na & 4,00 & .72008 & 4,20 & ,837 & 3,96 &, 706 & 4,22 & .441 & 3,89 & 809 \\
\hline Capacldade geral de resposta as mercado & 4,18] & ,77237 & 4,80 & ,447] & 4,04 & .767] & 4,11] & ,782 & 4,21] &, 787 \\
\hline
\end{tabular}

Fonte: Elaboração própria

Analisando-se a tabela 2, especificamente a coluna “Todos Hotéis", observou-se que a capacidade percebida pelos grandes hotéis portugueses de gerar informações de mercado é bastante alta (4,07 em média, que corresponde a 81,4\% do valor máximo), o mesmo acontecendo com a capacidade percebida de resposta ao mercado (média de 4,18, 83,6\% do valor máximo). Já a capacidade de disseminar as informações obtidas junto ao restante da empresa (um dos constructos que caracterizam a implementação do conceito de marketing nas organizações) foi inferior aos demais constructos, recebendo nota média de 3,71 (equivalente a 74,2\% do valor máximo). Quanto às variáveis, constatou-se:

- A pesquisa de mercado não houve um consenso entre os inquiridos, ou seja, há grupos de hotéis que realizam pouca ou nenhuma pesquisa de mercado (ou alguns dos profissionais que actuam no grupo não sabem da existência ou freqüência da realização destas pesquisas);

- A elevada dispersão de respostas na variável "pesquisa com clientes" reflete o desconhecimento de partes dos profissionais que actuam nestes grupos de hotéis, quanto a avaliação junto aos clientes da qualidade dos serviços oferecidos;

- Duas variáveis do constructo da disseminação de informações do mercado (referentes à divulgação para toda a empresa de problemas com clientes importantes e aos resultados da 
satisfação dos clientes) receberam valores médios abaixo do esperado (3,64, equivalente a $72,8 \%$ do valor máximo) e grande dispersão de respostas, o que reflecte que estas informações, em alguns dos grandes grupos hoteleiros, não são divulgadas adequadamente;

- Quanto ao constructo resposta ao mercado, também duas variáveis se destacaram com valor médios abaixo do esperado: "resposta a campanhas de comunicação de concorrentes" e “actividades coordenadas entre departamentos"; estas variáveis apresentaram-se como as mais frágeis quando se pretende responder ao mercado;

- Destaque também para a elevada dispersão de respostas em duas variáveis: "lentidão na resposta aos concorrentes” e "reclamações dos clientes não são ouvidas”; esta alta dispersão configura que parte dos indivíduos que actuam nestes grupos de hotéis desconhecem a velocidade de resposta da sua empresa perante aos concorrentes, bem como o tratamento das queixas de clientes.

Para aprofundar o entendimento sobre os resultados gerais, duas comparações foram realizadas: grupos estrangeiros versus grupos portugueses; profissionais de marketing versus profissionais de outros sectores. No primeiro comparativo, constatou-se que médias mais altas (para aspectos positivos) ou mais baixas (para aspectos negativos) foram predominantemente dos grupos estrangeiros. O mesmo ocorreu com as dispersões de respostas (desvio padrão), onde as menores dispersões foram constactadas nos grupos estrangeiros. Em relação aos grupos portugueses, encontrou-se médias inferiores aos estrangeiros e maior dispersão de respostas. Isto leva a concluir que a orientação para o mercado em grupos estrangeiros é mais evidente do que em grupos portugueses. Respostas muito variáveis demonstraram que na organização nem todos postulam do mesmo discurso.

No que tange a comparação entre profissionais do marketing e profissionais de outras áreas, constatou-se melhores resultados e menor dispersão de respostas entre os actuantes nas áreas de marketing das redes investigadas. Já os profissionais de outras áreas evidenciaram respostas mais dispersas, o que leva a crer que o discurso do departamento de marketing não é propriamente acompanhado pelos demais sectores da organização.

Em resumo, constatou-se uma boa capacidade de gerar informações de mercado (inteligência de marketing) e de responder ao mercado (maioria das respostas entre concordo totalmente e concordo parcialmente), mas a disseminação das informações coletadas não são um consenso 
(maioria das respostas em posição neutra) entre os profissionais que actuam nos diversos departamentos dos grandes grupos de hotéis de Portugal.

\subsection{Análises multivariadas}

Nesta etapa, a primeira análise concentrou-se em estabelecer as correlações entre as variáveis gerais da pesquisas (“Capacidade geral de gerar informações de mercado”; “Capacidade geral de disseminar as informações de mercado”; “Capacidade geral de resposta ao mercado”). Utilizou-se como ferramenta de apoio o software SPSS (Statistical Package for the Social Sciences).

Fazendo-se o teste de correlação de Pearson entre as três variáveis, encontrou-se uma correlação significativa entre a "capacidade geral de gerar informações para o mercado” e a “capacidade geral de resposta ao mercado”. O constructo "capacidade geral de disseminar as informações de mercado” não correlacionou-se com nenhum dos outros dois construtos, quando a base de dados foram todos os inquiridos. Separando-se as bases de dados (hotéis estrangeiros, hotéis portugueses, profissionais de marketing, profissionais de outras áreas), a única correlação significativa que surgiu com o constructo "capacidade geral de disseminar as informações de mercado” foi na base dos profissionais de marketing e a correlação deste constructo foi com o constructo “capacidade geral de resposta ao mercado”.

Outra análise multivariada utilizada foi a análise de clusters. Esta análise visou agrupar os casos semelhantes, evidenciando dois clusters: o cluster dos hotéis internacionais e o dos profissionais de marketing (grupo 1). Neste cluster, obteve-se melhores valores em todos os itens que mediram a orientação para o mercado dos maiores grupos de hotéis de Portugal. O outro cluster (grupo 2) caracterizou-se por agrupar hotéis de origem portuguesa e profissionais de áreas distintas ao marketing. Este segundo cluster evidenciou valores mais baixos em todos as variáveis, ou seja, menos orientado para o mercado (tabela 3). 
Tabela 3 - Análise de Clusters

\begin{tabular}{|c|c|c|c|c|c|c|c|c|c|}
\hline \multicolumn{2}{|c|}{ Ward Nethod } & $\begin{array}{c}\begin{array}{c}\text { Encontros periodicos com } \\
\text { clestes }\end{array} \\
\end{array}$ & Fesquasa de mercsso & \begin{tabular}{|l|} 
Lertisto na detecchlo de \\
rusanças de preterenclas
\end{tabular} & Fesquiss ).rito a clentes & $\begin{array}{l}\text { Lertidsto na deterchlo de } \\
\text { mudsrçse no metcado }\end{array}$ & 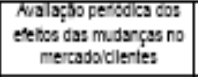 & $\begin{array}{l}\text { Cscacilade geral de gerar } \\
\text { irfomsçles do metcado }\end{array}$ & $\begin{array}{c}\text { Reuniles periodicas entre } \\
\text { desortamentos }\end{array}$ \\
\hline \multirow[t]{3}{*}{1} & Mean & 4,5 & 4,06 & 1,39 & 4,29 & 1,29 & \begin{tabular}{|c|c|} 
\\
\end{tabular} & 441 & 4,71 \\
\hline & $\mathrm{N}$ & 17 & 17 & 17 & 17 & 17 & 17 & 17 & 17 \\
\hline & Str. Deviaton &, 717 & ,748 & ,606 &, 772 & , 470 & A93 & ,618 & ,588 \\
\hline \multirow[t]{3}{*}{2} & Mean & 400 & 3,82 & 2,18 & 3,55 & 2,45 & 3,45 & 3,55 & 3,72 \\
\hline & $\mathrm{N}$ & 11 & 11 & 11 & 11 & 11 & 11 & 11 & 11 \\
\hline & Sar. Devation &,$\pi 5$ & 1,250 & ,982 & 1,292 & 688 & 1,036 & 934 & 1,009 \\
\hline \multirow[t]{3}{*}{ Total } & Mean & 4,32 & 3,96 & 1,68 & 4,00 & 1,79 & 4,18 & 4,07 & 4,32 \\
\hline & $\mathrm{N}$ & 28 & 28 & 28 & 28 & 28 & 28 & 28 & 28 \\
\hline & Sos. Deviaton &, 772 & ,962 & ,963 & 1,054 & ,799 & , 345 & ,858 & ,908 \\
\hline \multicolumn{2}{|c|}{ Ward Method } & $\begin{array}{l}\text { Dlacussto MeT/outros } \\
\text { cepbse necessidsdes } \\
\text { finuras dos clentes }\end{array}$ & $\begin{array}{c}\text { Probiemas com clertes } \\
\text { Impotartes traremlidas } \\
\text { rapicaments a todos }\end{array}$ & 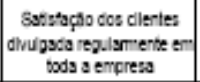 & $\begin{array}{l}\text { Lentidbo de ceptas } v \\
\text { comuricarem robre } \\
\text { questbes de mercado }\end{array}$ & 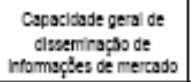 & $\begin{array}{l}\text { Lentdbo de regsoeta s } \\
\text { concorentes }\end{array}$ & $\begin{array}{c}\text { Irorar recorthesimento de } \\
\text { novss recessidases dos } \\
\text { clentes }\end{array}$ & $\begin{array}{l}\text { Revisto cortinua dos } \\
\text { serviços do hotal }\end{array}$ \\
\hline \multirow[t]{3}{*}{1} & Mean & 4,76 & 4,12 & 4,18 & 1,76 & 4,12 & 1,41 & 1,29 & 4,65 \\
\hline & $\mathrm{N}$ & 17 & 17 & 17 & 17 & 17 & 17 & 17 & 17 \\
\hline & Str. Devation & 437 & , 761 & ,951 & ,903 & , 73 & 1,004 & ,588 & ,606 \\
\hline \multirow[t]{3}{*}{2} & Mean & 3,00 & 2,9ा & 282 & 2,64 & 3,09 & 2,36 & 2,27 & 3,55 \\
\hline & $\mathrm{N}$ & 11 & 11 & 11 & 11 & 11 & 11 & 11 & 11 \\
\hline & sos. Deviation & 1,020 & 1,136 & 1,168 & ,924 & ,539 & ,924 & ,908 & ,688 \\
\hline \multirow[t]{3}{*}{ Tobal } & Mean & 4,07 & 3,84 & 3,64 & 2,11 & 3,71 & 1,79 & 1,68 & 4,21 \\
\hline & $\mathrm{N}$ & 28 & 28 & 28 & 28 & 28 & 28 & 28 & 28 \\
\hline & Srat. Deviaton & 1,120 & 1,056 & 1,224 & 984 & ,954 & 1,057 & 863 & ,833 \\
\hline \multicolumn{2}{|c|}{ Ward Method } & $\begin{array}{c}\text { Feurlbes de deptos para } \\
\text { responder as mudanças no } \\
\text { mercado }\end{array}$ & \begin{tabular}{|c} 
Resposis a camparhas de \\
comuricapho de \\
concornentss
\end{tabular} & $\begin{array}{l}\text { Afolissdes coordenstas } \\
\text { etre deptos }\end{array}$ & $\begin{array}{c}\text { Reciamsçles dos clentes } \\
\text { rJo sto ouvidas }\end{array}$ & $\begin{array}{l}\text { Incapscidase de colocar } \\
\text { em praticas um granse } \\
\text { plano de MKT }\end{array}$ & $\begin{array}{c}\text { Ao descobrit necessi dase } \\
\text { ce mudanças os daptos } \\
\text { realzam-na }\end{array}$ & $\begin{array}{l}\text { Caparitade geral de } \\
\text { resposta so mercaso }\end{array}$ & \\
\hline \multirow[t]{3}{*}{1} & Mean & \begin{tabular}{|r|}
43 \\
\end{tabular} & \begin{tabular}{|r|}
3,94 \\
\end{tabular} & 4,00 & 1,24 & 1,39 & 4,24 & 4,47 & \\
\hline & $\mathrm{N}$ & 17 & 17 & 17 & 17 & 17 & 17 & 17 & \\
\hline & sor. Devation & ,786 & 1,258 & , & ,970 &, 702 & 664 &, 717 & \\
\hline \multirow[t]{3}{*}{2} & Mean & 3,59 & 2,82 & 3,00 & 1,82 & 2009 & 3,64 & 3,73 & \\
\hline & $\mathrm{N}$ & 11 & 11 & 11 & 11 & 11 & 11 & 11 & \\
\hline & Sta. Deviation & ,688 & , 751 & 447 & 1,401 & , 344 &, 674 & ,647 & \\
\hline \multirow{3}{*}{ Tokal } & Mean & 4,04 & 3,50 & 3,51 & 1,46 & 1,64 & 4,00 & 4,18 & \\
\hline & $\mathrm{N}$ & 28 & 28 & 28 & 28 & 28 & 28 & 28 & \\
\hline & Sor. Deviation & 838 & 1,232 & ,786 & 1,170 &, 870 &, 720 &, 772 & \\
\hline
\end{tabular}

Fonte: SPSS versão 17.0

A última análise multivariada foi a regressão linear múltipla. Objectivou-se encontrar as variáveis que mais contribuem para os três constructos da escala Markor: "geração de informação de mercado” (6 variáveis independentes); “disseminação de informações do mercado” (5 variáveis independentes); e “resposta ao mercado” (9 variáveis independentes).

Para a realização desta análise, alguns testes de validade obrigatoriamente necessitam ser realizados. Os testes descritos na sequência foram efectuados:

- Os primeiros testes avaliaram três características fundamentais para validar a análise de regressão realizada: normalidade dos resíduos, variância constante dos resíduos e aleatoriedade dos resíduos. Os gráficos gerados no SPSS para as três regressões comprovaram a normalidade dos resíduos, a constância da variância dos resíduos e a aleatoriedade dos resíduos. Estes três factores validaram as análises de regressão efetuadas; 
- Em seguida, avaliaram-se os resultados do teste ANOVA. Nos dados gerados pelo SPSS, percebeu-se que os três modelos apresentados são úteis para explicar a relação entre cada uma das três variáveis dependentes e suas respectivas variáveis independentes. Considerando-se que a hipótese nula do teste de ANOVA é que o modelo não é útil, rejeitou-se a hipótese nula, pois a significância, nos três casos, foi inferior a 0,05.

Tendo-se a validez confirmada, partiu-se para a realização da regressão linear, utilizando-se o método stepwise. Os resultados obtidos para o construto "geração de informações de mercado” estão na tabela 4.

Tabela 4 - Regressão linear do construto geração de informações de mercado

\begin{tabular}{|c|c|c|c|c|c|}
\hline \multicolumn{6}{|c|}{ Coefficients $^{2}$} \\
\hline \multirow[b]{2}{*}{ Model } & \multicolumn{2}{|c|}{ Coefficients } & \multirow{2}{*}{$\begin{array}{c}\text { Standardized Coefficients } \\
\text { Beta }\end{array}$} & \multirow[b]{2}{*}{$t$} & \multirow[b]{2}{*}{ Sig. } \\
\hline & \multicolumn{2}{|c|}{\begin{tabular}{l|l|} 
B & Std. Error
\end{tabular}} & & & \\
\hline 1 (Constant) & 4,158 & .574 & \multirow{3}{*}{$\begin{array}{r}-.584 \\
.317\end{array}$} & 7,238 & .000 \\
\hline Lentidäo na deteç̧ão de mudanças no mercado &,- 637 & 155 & & $-4,121$ & .000 \\
\hline \multirow[t]{4}{*}{ Pesquisa junto a clientes } & .258 & .117 & & 2,198 & 037 \\
\hline & \multicolumn{5}{|c|}{ Model Summary ${ }^{\circ}$} \\
\hline & Model & $\mathrm{R}$ & R Square & Adjusted R Square & Std. Error of the Estimate \\
\hline & &, $697^{\circ}$ & .488 & \begin{tabular}{r|}
.444 \\
\end{tabular} & .838 \\
\hline
\end{tabular}

Fonte: SPSS versão 17.0

Observando-se a tabela 4, percebeu-se que o $\mathrm{R}^{2}$ ajustado, que demonstra quanto as variáveis independentes explicam a variância da variável dependente, ficou em 44,4\%, um bom ajustamento do modelo. Assim, pode ajustar-se a equação de regressão, onde somente as variáveis “lentidão na detecção de mudanças no mercado" e "pesquisas junto a clientes” se mostraram significativas, pois a sua significância foi menor do que 0,05 (tabela 4, última coluna). As demais variáveis mostraram-se não significativas. Desta forma, a equação de regressão foi determinada ao realizar-se novamente a regressão com apenas as variáveis significativas, chegando-se assim a seguinte equação: Geração de informações de mercado = 4,156 - 0,637*Lentidão na detecção de mudanças no mercado + 0,258*Pesquisa junto a clientes.

Esta equação representa que cada incremento negativo (sinal negativo) de uma unidade da variável “lentidão na detecção de mudanças no mercado”, incrementa positivamente em 0,637 a capacidade de geração de informações de mercado. O mesmo raciocínio utiliza-se para a 
variável "pesquisas junto a clientes", onde o incremento positivo de uma unidade desta variável incrementa positivamente em 0,258 a geração de informações de mercado.

Na seqüência, para o constructo “disseminação de informações de mercado”, os resultados da regressão foram apresentados na tabela 5.

Tabela 5 - Regressão linear do construto disseminação de informações de mercado

\begin{tabular}{|c|c|c|c|c|c|}
\hline \multicolumn{6}{|c|}{ Coefficients $^{2}$} \\
\hline \multirow[b]{2}{*}{ Model } & \multicolumn{2}{|c|}{\begin{tabular}{l|l} 
Coefficients & \\
\end{tabular}} & \multirow{2}{*}{\begin{tabular}{|c|} 
Standardized Coeficients \\
Beta
\end{tabular}} & \multirow[b]{2}{*}{$t$} & \multirow[b]{2}{*}{ Sig. } \\
\hline & B & Sto. Error & & & \\
\hline 1 (Constant) & .870 & .801 & & 1,116 & .025 \\
\hline $\begin{array}{l}\text { Discussäo entre MKT e outros deptos sobre necessidades futuras dos } \\
\text { clientes }\end{array}$ & .354 & .117 & .484 & 3,033 & .006 \\
\hline \multirow[t]{4}{*}{ Reuniồes periódicas entre departamentos } & .371 &, 144 &, 393 & 2,565 & .017 \\
\hline & \multicolumn{5}{|c|}{ Model Summary } \\
\hline & Model & $\mathrm{R}$ & R Square & Adjusted R Square & Std. Emor of the Estimate \\
\hline & 1 & $.731^{\circ}$ & ,534 & .497 & ,606 \\
\hline
\end{tabular}

Fonte: SPSS versão 17.0

Analisando-se a tabela 5, observa-se que o $\mathrm{R}^{2}$ ajustado ficou em 49,7\%, também um bom ajustamento do modelo. Neste caso somente as variáveis “discussão MKT/outros deptos sobre necessidades futuras dos clientes” e “reuniões periódicas entre departamentos” se mostraram significativas, pois a sua significância foi menor do que 0,05 (tabela 5, última coluna). As demais variáveis mostraram-se não significativas. Sendo assim, a equação de regressão foi determinada ao realizar-se novamente a regressão com apenas as variáveis significativas, chegando-se assim a seguinte equação: Disseminação de informações de mercado $=0,670+$ 0,354*Discussão MKT/outros deptos sobre necessidades futuras dos clientes + 0,371*Reuniões periódicas entre departamentos.

Portanto, para cada incremento positivo de uma unidade da variável “discussão MKT/outros deptos sobre necessidades futuras dos clientes", incrementa positivamente em 0,354 a capacidade de disseminação de informações de mercado. O mesmo raciocínio utiliza-se para a variável "reuniões periódicas entre departamentos", onde o incremento de uma unidade desta variável incrementa em 0,371 a disseminação de informações de mercado.

Por fim, para o constructo "resposta ao mercado", os resultados da regressão foram apresentados na tabela 6 . 
Tabela 6 - Regressão linear do construto resposta ao mercado

\begin{tabular}{|c|c|c|c|c|c|}
\hline \multicolumn{6}{|c|}{ Coefficients $^{2}$} \\
\hline \multirow[b]{2}{*}{ Model } & \multicolumn{2}{|c|}{ Coefficients } & Standardized Coefficients & \multirow[b]{2}{*}{$\mathrm{t}$} & \multirow[b]{2}{*}{ Sig. } \\
\hline & $\mathrm{B}$ & Std. Error & Beta & & \\
\hline 1 (Constant) & 1,807 & .528 & & 3,420 &, 002 \\
\hline \multirow[t]{4}{*}{ Atividades coordenadas entre deptos } &, 657 &, 143 &, 689 & 4,589 &, 000 \\
\hline & \multicolumn{5}{|c|}{ Model Summary } \\
\hline & Model & $R$ & R Square & Adjusted R Square & Std. Error of the Estimate \\
\hline & 1 & $.689^{9}$ & ,447 & ,426 & ,585 \\
\hline
\end{tabular}

Fonte: SPSS versão 17.0

Neste último constructo, o $\mathrm{R}^{2}$ ajustado resultou em 42,6\%, bom ajustamento do modelo (tabela 6). A análise efetuada identificou somente uma variável significativa ("actividades coordenadas entre deptos”). Realizando-se novamente a regressão com apenas a variável significativa, obteve-se a seguinte equação: Resposta ao mercado $=1,807+0,657 *$ Atividades coordenadas entre deptos.

Conforme o mesmo raciocínio das regressões anteriores, um incremento na variável “atividades coordenadas entre deptos” incrementa positivamente em 0,657 a capacidade de resposta ao mercado. Esta constatação encerrou a fase das análises dos dados obtidos na investigação.

\section{Conclusões e Recomendações}

$\mathrm{Na}$ actualidade, o marketing mostra-se relevante tanto para organizações quanto para a sociedade (KOTLER, 2005). A evolução deste importante conceito, que inicialmente buscava facilitar as transações entre compradores e vendedores, chegou a um ponto de posicionar-se como a principal função dentro das empresas, sendo considerado uma filosofia de actuação organizacional (BROWN, 1987; NARVER; SLATER, 1990).

Considerando a importância do marketing para o meio empresarial, o perfeito entendimento e a aplicação do seu conceito por todos os envolvidos numa organização torna-se fundamental para o sucesso de uma empresa (LAFFERTY; HULT, 2001). É o que Kohli e Jaworski (1990) chamaram de orientação para o mercado.

Neste estudo, como objectivo geral, buscou-se medir o grau de orientação para o mercado dos principais representantes de um importante sector para a economia portuguesa: o segmento 
hoteleiro. A partir de uma investigação empírica com 17 dos 20 principais grupos de hotéis de Portugal, e utilizando-se como instrumento de coleta de dados a tradicional escala Markor (KOHLI; JAWORSKI, 1990), constatou-se que tanto a geração de informações de mercado (inteligência de marketing), quanto a capacidade de resposta ao mercado, são bem percebidos pelos profissionais que actuam na chefia das diversas áreas dos grupos hoteleiros investigados. Porém, observou-se que a disseminação das informações obtidas junto ao mercado, um dos constructos da orientação para o mercado, carece de uma maior atenção, afinal, a investigação empírica demonstrou que nem todos os colaboradores pesquisados têm noção das informações geradas, pois este constructo não correlacionou-se com os dois outros constructos (geração de informações de mercado e resposta ao mercado, estes dois correlacionados entre si). Conclui-se desta forma que a implementação do conceito de marketing nas grandes redes de hotéis de Portugal, traduzido como orientação para o mercado, pode ser considerada, mas para isso precisa de ser melhor trabalhada internamente pelos gestores destas organizações, em especial pleos executivos responsáveis pelo marketing destes grupos hoteleiros.

Em termos de objectivos específicos, o primeiro tratava da comparação entre hotéis de origem estrangeira versus hotéis genuinamente portugueses. Constatou-se nas análises descritivas e de clusters que hotéis estrangeiros tendem a serem mais orientados para o mercado do que os hotéis nacionais. Comparação semelhante, porém entre as funções exercidas pelos inquiridos, sendo o segundo objectivo específico deste estudo. Pode-se constatar que os profissionais de marketing dos grandes grupos hoteleiros de Portugal tendem a ser orientados para o mercado, porém o mesmo não acontece em toda a organização, afinal uma grande parte dos colaboradores das empresas investigadas, que actuam em funções distintas ao marketing, não demonstraram a mesma orientação para o mercado dos profissionais de marketing destas empresas.

O último objectivo específico era identificar quais os elementos que são significativos para a optimização dos graus de geração e disseminação de informações de mercado e de resposta ao mercado dos grandes grupos de hotéis presentes em Portugal. Verificou-se que, para melhorar a geração de informações de mercado nestas organizações, é necessário ser rápido na detecção de mudanças no mercado e continuamente realizar mais e melhores pesquisas junto aos clientes. No que tange a disseminação das informações de mercado, há a necessidade do departamento de marketing destes grupos de hotéis discutirem com os outros departamentos as necessidades futuras dos clientes, sendo que estas reuniões devem ser periódicas. Por fim, 
quanto ao grau de resposta ao mercado, o indicador mais importante é o trabalho conjunto entre os departamentos. Estes factores elencados aqui contribuem significativamente para uma melhor orientação de mercado dos grandes grupos hoteleiros de Portugal, implementando definitivamente o conceito de marketing nestas organizações.

Como implicações ao estudo realizado, ficou registada a importância do conceito de marketing para as principais organizações que actuam no sector de hotelaria em Portugal, bem como os pontos a melhorar para atingir um alto grau de orientação para este importante mercado. Em termos acadêmicos, ressaltou-se a relevância da disciplina do marketing, considerando a aplicabilidade das suas teorias na realidade social. Investigações científicas no campo do marketing surgem como fundamentais para o desenvolvimento organizacional e social.

Em termos de limitações, importa ressaltar que a pesquisa focalizou somente os maiores representantes de um sector relevante da economia portuguesa: o sector de hotelaria em Portugal. Não foram consideradas as pequenas e médias empresas deste sector, portanto não foi realizada uma amostragem estatisticamente significativa, o que impede a generalização dos resultados e o estabelecimento de um grau geral de orientação para o mercado dos hotéis portugueses. Desta forma, como recomendações de estudos futuros, sugere-se a ampliação da mesma pesquisa para todo o sector hoteleiro português, bem como a mensuração da orientação para o mercado de outros importantes sectores da economia de Portugal. Esta ampla pesquisa pode auxiliar no desenvolvimento de futuros projectos de ampliação da competitividade da nação portuguesa.

\section{Referências}

ATLAS DA HOTELARIA. 2009. Publituris Hotelaria. ed. 01-03-2009, pp. 30-36.

BAKER, M. 2003. The Marketing Book. 5 ed. Oxford: Elsevier Science.

BARTELS, R. 1988. The History of Marketing Thought. Columbus: Publishing Horizons.

BROWN, R. 1987. Marketing: a Function and a Philosophy. The Quarterly Review of Marketing. Spring/Summer, pp.25-30.

COOPER, C.; FLETCHER, J.; FYALL, A.; GILBERT, D.; WANHILL, S. 2008. Tourism: Principles and Practice. 3 Ed. Harlow. England: Prentice Hall Inc.

DAY, G. 1994. The Capabilities of Market Driven Organizations. Journal of Marketing. Vol. 58, October, pp.37-52.

DESHPANDÉ, R.; WEBSTER, F. 1989. Organizational Culture and Marketing: Defining the Research Agenda. Journal of Marketing. Vol.53, January, pp. 3-15. 
.; FARLEY, J.; WEBSTER, F. 1993. Corporate Culture, Customer Orientation and Innovativeness in Japonese Firms: a Quadrad Analysis. Journal of Marketing. Vol. 57, January, pp. 23-37.

FOLEY, A.; FAHY, J. 2009. Seeing Market Orientation Through a Capabilities Lens. European Journal of Marketing. Vol. 43, $\mathrm{n}^{\circ}$ 1/2, pp. 13-20.

GREENLEY, G.; HOOLEY, G.; SAUNDERS, J. 2004. Management Processes in Marketing Planning. European Journal of Marketing. Vol. 38, n 8, pp. 933-955.

GRÖNROOS, C. 1989. Defining Marketing: a Market-Oriented Approach. European Journal of Marketing. Vol. 23, n 1, pp.52-60.

HAIR JR. J. F.; BABIN, B.; MONEY, A. H.; SAMOUEL, P. 2003. Essentials of business research methods. New Jersey: John Wiley \& Sons.

HOWARD, J. 1983. Theory of the Firm. Journal of Marketing. Vol.47, October, pp.90-100.

JAWORSKI, B.; KOHLI, A. 1993. Market Orientation: Antecedents and Consequences. Journal of Marketing. Vol. 57, July, pp.53-70.

JOCZ, K.; QUELCH, J. 2008. An Exploration of Marketing's Impacts on Society: a Perspective Linked to Democracy. Journal of Public Policy \& Marketing. Vol. 27, n² 2, pp. 202-206.

KOHLI, A.; JAWORSKI, B. 1990. Market Oientation: the Construct, Research Propositions and Managerial Applications. Journal of Marketing. Vol. 54, April, pp. 1-18.

; ; _ .; KUMAR, A. 1993. MARKOR: a Mensure of Market Orientation. Journal of Marketing Research. Vol.32, November, pp. 467-477.

KOLLER, L. 2002. Market Orientation in the REFAP S.A. Refinery: a Exploratory Study. Master's thesis. FederalUniversity of Rio Grande do Sul, Porto Alegre, Brazil.

KOTLER, P. 2005. The Role Played by the Broadening of Marketing Movement in the History of Marketing Thought. Journal of Public Policy \& Marketing. Vol. 24, n 1, pp. 114-116.

Prentice-Hall Inc.

; ARMSTRONG, C. 1996. Principles of Marketing. 7 ed. Englewood Cliffs, New Jersey: ; KELLER, K. 2005. Marketing Management. 12 ed. New Jersey: Pearson-Prentice Hall.

Janeiro, pp. 10-15.

LAFFERTY, B.; HULT, G. 2001. A Synthesis of Contemporary Market Orientation Perspectives. European Journal of Marketing. Vol. 35, nº 1/2, pp. 92-109.

LEVITT, T. 1986. The Marketing Imagination. New York: Free Press.

McKENNA, R. 1991. Relationship Marketing: Successful Strategies for the Age of the Customer. New York: Addison Wesley.

NARVER, J.; SLATER, S. 1990. The Effect of a Market Orientation on Business Profitability. Journal of Marketing. Vol. 54, pp. 20-35.

PEARSON, A. 2002. Tough-Minded Ways to Get Innovative. The Innovative Enterprise in Harvard Business Review. Special issue, August, pp. 117-124.

PITER. 2005. Land of Vouga and Caramulo: Dynamise Tourism in Mountain Areas. PITER Integrated Program of Nature Tourism of Regional and Structuring Base. Portaria $n^{\circ}$ 450/2001, May 5 .

RAIIJ, E.; STOELHORST, J. 2008. The Implementation of a Market Orientation. European Journal of Marketing. Vol. 42, n 11/12, pp. 1265-1293. 
THOMAS, M. 2006. The malpractice of marketing management. Marketing Intelligence \& Planning. Vol. 24, nº 2, pp.96-101.

WILKIE, W.; MOORE, E. 2007. What Does the Definition of Marketing Tell us About Ourselves? Journal of Public Policy \& Marketing. Vol. 26, n² 2, pp. 269-276.

Recebido em: 10/08/2009

Aprovado em: 30/11/2009 